\title{
Corrigendum: Herbal medicines in Brazil: pharmacokinetic profile and potential herb-drug interactions
}

\author{
Andre L. D. A. Mazzari and Jose M. Prieto* \\ Pharmacognosy, School of Pharmacy, University College London, London, UK \\ *Correspondence: j.prieto@ucl.ac.uk \\ Edited by: \\ Adejuwon Adewale Adeneye, Lagos State University College of Medicine, Nigeria \\ Reviewed by: \\ Fang-Rong Chang, Kaohsiung Medical University, Taiwan \\ Eleni Skaltsa, National and Kapodistrian University of Athens, Greece
}

Keywords: herb-drug interactions, cytochrome P450, glutathione, glucuronidation, P-glycoprotein, polymorphism, Brazil, pharmacovigilance

\section{A corrigendum on}

Herbal medicines in Brazil: pharmacokinetic profile and potential herb-drug interactions

by Mazzari, A. L. D. A., and Prieto, J. M. (2014). Front. Pharmacol. 5:162. doi: 10.3389/fphar.2014.00162

We noticed that some symbols were lacking in Table 5 and some of the Plant Family names were not currently accepted.
We hereby present the Tables 1-9 with all symbols and botanical names re-checked.

Apologies for any inconvenience this may have caused.

Conflict of Interest Statement: The authors declare that the research was conducted in the absence of any commercial or financial relationships that could be construed as a potential conflict of interest.

Received: 07 November 2014; accepted: 27 January 2015; published online: 10 March 2015.
Citation: Mazzari ALDA and Prieto JM (2015) Corrigendum: Herbal medicines in Brazil: pharmacokinetic profile and potential herb-drug interactions. Front. Pharmacol. 6:23. doi: 10.3389/fphar.2015.00023

This article was submitted to Ethnopharmacology, a section of the journal Frontiers in Pharmacology.

Copyright (๑) 2015 Mazzari and Prieto. This is an openaccess article distributed under the terms of the Creative Commons Attribution License (CC BY). The use, distribution or reproduction in other forums is permitted, provided the original author(s) or licensor are credited and that the original publication in this journal is cited, in accordance with accepted academic practice. No use, distribution or reproduction is permitted which does not comply with these terms.

\section{Table 1 | Medicinal plant species listed in RENISUS with reported effects of on CYP1A2.}

\begin{tabular}{|c|c|c|}
\hline Plant species/Family & Effects on CYP1A2 & References \\
\hline Curcuma longa (Zingiberaceae) & + & Thapliyal et al., 2002 \\
\hline Glycine max (Leguminosae) & - & Shon and Nam, 2004 \\
\hline Harpagophytum procumbens (Pedaliaceae) & $\mathrm{NE},-$ & Unger and Frank, 2004; Modarai et al., 2011 \\
\hline Mentha piperita (Lamiaceae) & - & Unger and Frank, 2004 \\
\hline Punica granatum (Lythraceae) & - & Faria et al., 2007a \\
\hline Trifolium pratense (Leguminosae) & - & Unger and Frank, 2004 \\
\hline
\end{tabular}

+ , Enzyme induction; -, Enzyme inhibition; NE, No Effect.

Table 2 | Medicinal plant species listed in RENISUS with reported effects of on CYP2C9.

\begin{tabular}{lcc}
\hline Plant species/Family & Effects on CYP2C9 & References \\
\hline Allium sativum (Amaryllidaceae) &,-+ & Foster et al., 2001; Ho et al., 2010 \\
Eucalyptus globulus (Myrtaceae) & - & Unger and Frank, 2004 \\
Harpagophytum procumbens (Pedaliaceae) & NE, & Modarai et al., 2011 \\
Mentha piperita (Lamiaceae) & - & Unger and Frank, 2004 \\
Punica granatum (Lythraceae) & - & Hanley et al., 2012 \\
Trifolium pratense (Leguminosae) & - & Unger and Frank, 2004 \\
Zingiber officinale (Zingiberaceae) & - & Kimura et al., 2010
\end{tabular}

+, Enzyme induction; -, Enzyme inhibition; NE, No Effect. 
Table 3 | Medicinal plant species listed in RENISUS with reported effects of on CYP2C19.

\begin{tabular}{lcc}
\hline Plant species/Family & Effects on CYP2C19 & References \\
\hline Allium sativum (Amaryllidaceae) & - & Foster et al., 2001 \\
Eucalyptus globulus (Myrtaceae) & - & Unger and Frank, 2004 \\
Harpagophytum procumbens (Pedaliaceae) & NE & Modarai et al., 2011 \\
Mentha piperita (Lamiaceae) & - & Unger and Frank, 2004 \\
Trifolium pratense (Leguminosae) & - & Unger and Frank, 2004 \\
\hline
\end{tabular}

+ , Enzyme induction; -, Enzyme inhibition; NE, No Effect.

Table 4 | Medicinal plant species listed in RENISUS with reported effects of on CYP2D6.

Plant species/Family

Allium sativum (Amaryllidaceae)

Eucalyptus globulus (Myrtaceae)

Harpagophytum procumbens (Pedaliaceae)

Mentha piperita (Lamiaceae)

Phyllanthus amarus (Phyllanthaceae)

Punica granatum (Lythraceae)

Trifolium pratense (Leguminosae)

+, Enzyme induction; -, Enzyme inhibition; NE, No Effect.

\section{Effects on CYP2D6}

NE

$-$

NE, -

$-$

$-$

$-$

\section{References}

Markowitz et al., 2003

Unger and Frank, 2004

Modarai et al., 2011

Unger and Frank, 2004

Hari Kumar and Kuttan, 2006

Usia et al., 2006

Unger and Frank, 2004

\section{Table 5 | Medicinal plant species listed in RENISUS with reported effects of on CYP2E1.}

\section{Plant species/Family}

Allium sativum (Amaryllidaceae)

Curcuma longa (Zingiberaceae)

Glycine max (Leguminosae)

Momordica charantia (Cucurbitaceae)

Phyllanthus amarus (Phyllanthaceae)

Phyllanthus urinaria (Phyllanthaceae)

Punica granatum (Lythraceae)

\section{Effects on CYP2E1}

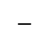

NE

NE

$-$

$-$

$-$

$-$

\section{References}

Le Bon et al., 2003

Salama et al., 2013

Shon and Nam, 2004

Raza et al., 1996

Hari Kumar and Kuttan, 2006

Shen et al., 2008

Faria et al., 2007a

+, Enzyme induction; -, Enzyme inhibition; NE, No Effect.

Table 6 | Medicinal plant species listed in RENISUS with reported effects of on CYP3A.

Plant species/Family

Allium sativum (Amaryllidaceae)

Chamomilla recutita (Compositae)

Curcuma longa (Zingiberaceae)

Eucalyptus globulus (Myrtaceae)

Foeniculum vulgare (Apiaceae)

Harpagophytum procumbens (Pedaliaceae)

Mentha piperita (Lamiaceae)

Momordica charantia (Cucurbitaceae)

Phyllanthus amarus (Phyllanthaceae)

Punica granatum (Lythraceae)

Trifolium pratense (Leguminosae)

Uncaria tomentosa (Rubiaceae)

Zingiber officinale (Zingiberaceae)

\section{Effects on CYP3A}

$$
\begin{aligned}
& \mathrm{NE},-\left(*{ }^{* *} /{ }^{* * *}\right) \\
& -\left(^{*}\right) \\
& \mathrm{NE}\left({ }^{*}\right) \\
& -\left(^{*}\right) \\
& -\left(^{*}\right) \\
& \mathrm{NE},-^{(*)} \\
& -\left(^{*}\right) \\
& -\left(^{*}\right) \\
& -\left({ }^{*} / * * * * *\right) \\
& -(* / * * * * *) \\
& -\left(^{*}\right) \\
& -\left(^{*}\right)
\end{aligned}
$$

\section{References}

Foster et al., 2001; Hajda et al., 2010

Budzinski et al., 2000

Graber-Maier et al., 2010

Unger and Frank, 2004

Subehan et al., 2006, 2007

Unger and Frank, 2004; Modarai et al., 2011

Unger and Frank, 2004

Raza et al., 1996

Hari Kumar and Kuttan, 2006

Faria et al., 2007a

Budzinski et al., 2000

Budzinski et al., 2000

Kimura et al., 2010

${ }^{*}$ CYP3A4,${ }^{* *}$ CYP3A5, ${ }^{* * *}$ CYP3A7/+, Enzyme induction; - Enzyme inhibition; NE, No Effect. 
Table 7 | Medicinal plant species listed in RENISUS with reported effects of on glutathione levels.

\begin{tabular}{|c|c|c|}
\hline Plant species/Family & Effects on glutathione levels & References \\
\hline Allium sativum (Amaryllidaceae) & + & Ip and Lisk, 1997 \\
\hline Anacardium occidentale (Anacardiaceae) & + & Singh et al., 2004 \\
\hline Baccharis trimera (Compositae) & - & Nogueira et al., 2011 \\
\hline Bauhinia forficata (Leguminosae) & - & Damasceno et al., 2004 \\
\hline Calendula officinalis (Compositae) & + & Preethi and Kuttan, 2009 \\
\hline Chamomilla recutita (Compositae) & + & Al-Hashem, 2010 \\
\hline Croton cajucara (Euphorbiaceae) & + & Rabelo et al., 2010 \\
\hline Curcuma longa (Zingiberaceae) & + & Rong et al., 2012 \\
\hline Cynara scolymus (Compositae) &,$+ N E$ & Miccadei et al., 2008 \\
\hline Mikania glomerata (Asteraceae) & NE & Barbosa et al., 2012 \\
\hline Momordica charantia (Cucurbitaceae) & + & Raza et al., 1996; Raza et al., 2000 \\
\hline Phyllanthus amarus (Phyllanthaceae) & + & Kumar and Kuttan, 2004, 2005; Karuna et al., 2009; Maity et al., 2013 \\
\hline Phyllanthus niruri (Phyllanthaceae) & + & Bhattacharjee and Sil, 2006; Manjrekar et al., 2008 \\
\hline Psidium guajava (Myrtaceae) & + & Tandon et al., 2012 \\
\hline Punica granatum (Lythraceae) &,+- & Dassprakash et al., 2012; Faria et al., 2007b \\
\hline Ruta graveolens (Rutaceae) & + & Ratheesh et al., 2011 \\
\hline Zingiber officinale (Zingiberaceae) &,$+ N E$ & Ajith et al., 2007 \\
\hline
\end{tabular}

+, Enzyme induction; -, Enzyme inhibition; NE, No Effect.

Table 8 | Medicinal plant species listed in RENISUS with reported effects of on UGT levels.

Plant species/Family

Allium sativum (Amaryllidaceae)

Curcuma longa (Zingiberaceae)

+, Enzyme induction;-, Enzyme inhibition; NE, No Effect.

\section{Effects on UGT levels}

\section{References}

Ip and Lisk, 1997

Naganuma et al., 2006

Table 9 | Medicinal plant species listed in RENISUS with reported effects of on P-glycoprotein activity.

Plant species/Family

Achillea millefolium (Compositae)

Allium sativum (Amaryllidaceae)

Curcuma longa (Zingiberaceae)

\section{Effects on P-glycoprotein activity}

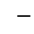

$+$

NE

\section{References}

Haidara et al., 2006

Hajda et al., 2010

Graber-Maier et al., 2010

+ , Efflux increase; -, Efflux decrease. 